\title{
INTEGRAL in HEAVENS
}

\section{R. Walter*, R. Rohlfs, M. Meharga, P. Binko, N. Morisset, M. Beck, N. Produit, L. Pavan, V. Savchenko, C. Ferrigno}

INTEGRAL Science Data Centre, Université de Genève, Chemin d'Ecogia 16, CH-1290 Versoix, Switzerland

E-mail: Roland.Walter@unige.ch

\section{A. Frankowski}

Centrum Astronomiczne im. M. Kopernika, Rabiańska 8, PL-87-100 Toruń, Poland

INTEGRAL Science Data Centre, Université de Genève, Chemin d'Ecogia 16, CH-1290 Versoix,

Switzerland

\section{P. Bordas}

Institut für Astronomie und Astrophysik, Universität Tübingen, Sand 1, 72076 Tübingen,

Germany

INTEGRAL Science Data Centre, Université de Genève, Chemin d'Ecogia 16, CH-1290 Versoix,

Switzerland

\begin{abstract}
With their large fields of view, INTEGRAL's instruments accumulate an enormous exposure time on any position of the sky. Collecting the scientific results of INTEGRAL for a given source requires downloading large amounts of data and installing and mastering the OSA analysis software package. This barrier is often too large for an archive user lacking detailed mission specific knowledge. The HEAVENS web interface provides on-the-fly remote analysis of INTEGRAL data and allows to generate high-level products customized for specific scientific needs.
\end{abstract}

8th INTEGRAL Workshop, The Restless Gamma-ray Universe, Integral2010,

September 27-30, 2010

Dublin Ireland

${ }^{*}$ Speaker. 


\section{Introduction}

The success of a research infrastructure is not only measured by the publications it produces but also by the information it makes available to the wider community. High-energy astronomy space missions have pioneered and demonstrated how powerful legacy data sets can be for generating new discoveries, especially when combined from many research infrastructures and analysed in ways the original researchers could not have anticipated.

Even if many data are available on-line, high-energy space mission archives face several challenges: (1) Data from the early missions, where they still exist, can no longer be re-analyzed, limiting the possibility for new data exploitation; (2) The detailed analysis know-how vanishes within a few years after the end of a mission, limiting the possibility for additional deep analyses as often triggered by the later missions; the number and variety of space missions and instruments is such that scientists do not have the resources and the knowledge to find and exploit efficiently all the data they have access to; (3) All astrophysical sources require a multi-wavelength approach. Scientists from lower energy regimes or theoretical fields need to access high-energy data easily, without barriers, to create new knowledge. Currently these communities have little chance of efficiently exploiting the data, especially in the post operation phase; (4) If the archived data cannot be analyzed straightforwardly, the public and the higher-level educators/students will, in particular, be least able to benefit from them. The data, the missions and the science results loose visibility with time. New scientific results become therefore difficult to extract even when very valuable data do exist on specific astronomical objects. The paradigm of distributing data and analysis software is working very well during mission operation but proves to be insufficient a few years later when the deep specific knowledge vanishes.

HEAVENS aims at improving this situation by providing not only data and software but analysis services for a number of recent and important high-energy missions. These services allow any user to perform on-the-fly analysis of the data to produce straightforwardly scientific results for any sky position, time and energy intervals without requiring mission specific software or instrumental knowledge. The ultimate goal is to ensure that the data of the present space missions could be effectively used by everyone and everywhere for the decades to come.

By providing a straightforward interface to complex data and data analysis, HEAVENS makes the data and the process of generating science products available to the public and to higher education, promoting the visibility of the efforts in space sciences to the society and encouraging the public to actively explore the data.

\section{Architecture and User interface}

The HEAVENS infrastructure consists of three components: (1) the "data servers" receiving specific data requests and providing on-the-fly data products generated on purpose or precalculated, (2) the "Web application", an augmented web client exploiting extended query facilities (using the Javascript framework JQuery and AJAX) and the data products and (3) a multiobservatory server dispatching requests between the "Web application", "Virtual Observatory clients" and the "data servers" and implementing caching and rendering of products. 
The INTEGRAL data are currently served by three types of data servers. The first type is serving products generated from a pixel archive (used for ISGRI and JEM-X). The second type, handling requests for time series, is used for OMC, SPI-ACS and IREM. A third type is serving pre-calculated products and currently used for PICsIT and SPI products.

Virtual Observatory clients can access the HEAVENS data products through the dispatcher. The dispatcher can easily be interfaced to external Virtual Observatory servers, extending the data that could be displayed through the HEAVENS interface.

\section{INTEGRAL data}

INTEGRAL [1] was launched on October 17, 2002 and will operate for more than 10 years. The INTEGRAL science payload consists of two main instruments, the spectrometer SPI and the imager IBIS (with the ISGRI and PICsIT detector layers) supplemented by two subsidiary instruments, the X-ray monitor JEM-X and the optical monitoring camera OMC. These four instruments have (fully-coded) fields of view of 16, 9, 4.8 and 5 degrees respectively. In addition INTEGRAL carry on the SPI anti-coincidence, the most sensitive all-sky soft gamma-ray detector flying and the local particle radiation environment monitor IREM. INTEGRAL observations are split in many short (typically $20 \mathrm{~min}$ ) pointings science windows, most of the time implementing a dithering pattern to optimize the image reconstruction and spatial resolution.

INTEGRAL data are processed at the ISDC [2] with the Offline Scientific Analysis ${ }^{1}$ (OSA) software. The data products are organized in a specific data archive to be used by various HEAVENS data servers. The processing of the data is described in further details in the next subsections.

\subsection{ISGRI and JEM-X products}

ISGRI [3, 4] and JEM-X [5] data are available in HEAVENS for the full mission. High-level science products, i.e. images, lightcurves and spectra, are built on request for user specified sky position, energy bands and sets of time intervals. The products are generated from a pixel database containing for each sky pixel and pointing science window (identified by start time and duration), the count rate, variance and effective exposure measured in a number of predefined energy bands. These values have been derived from the sky images reconstructed with the OSA software using an optimized point source catalogue for the image cleaning. Some quality information (e.g. measure of the background fluctuation) are also stored in the database to allow data selection based on their quality. The sky is divided in 6 overlapping regions each of them pixellized using a stereographic projection. The pixels are smaller than the instrument resolution. The OSA images are oversampled with a bicubic interpolation algorithm before being mapped to the predefined sky pixels

The pixel database is stored in binary files. Each file contains the history of all measurements for a predefined set of sky pixels covering the entire sky. When triggered, the HEAVENS INTEGRAL "data server" extracts the measurements from the database according to the user's specifications and build data products on-the-fly. Mosaic images are created by weighted average of the measured counts. Spectra and lightcurves are derived by fitting images built for each time/energy bin. The fitting is performed with a two dimensional point spread function fixed in position and

\footnotetext{
${ }^{1}$ available at http://www.isdc.unige.ch/integral/analysis
} 


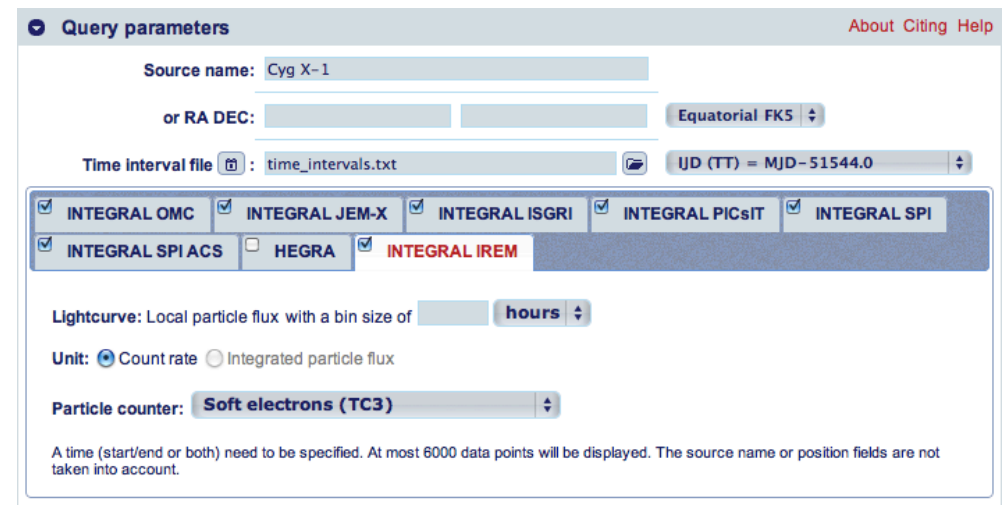

Submit Reset

- Cyg X-1 - High-Mass X-Ray Binary supergiant, black hole

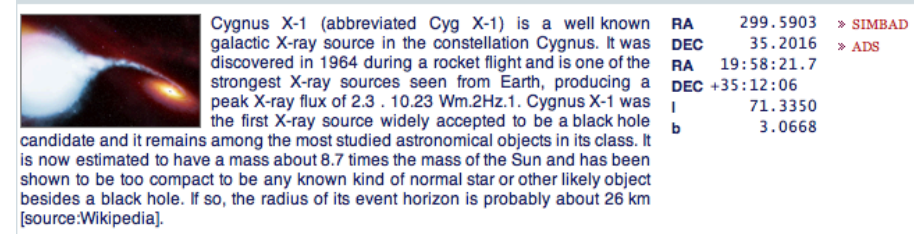
besides a black hole: Wikipedia].
[source

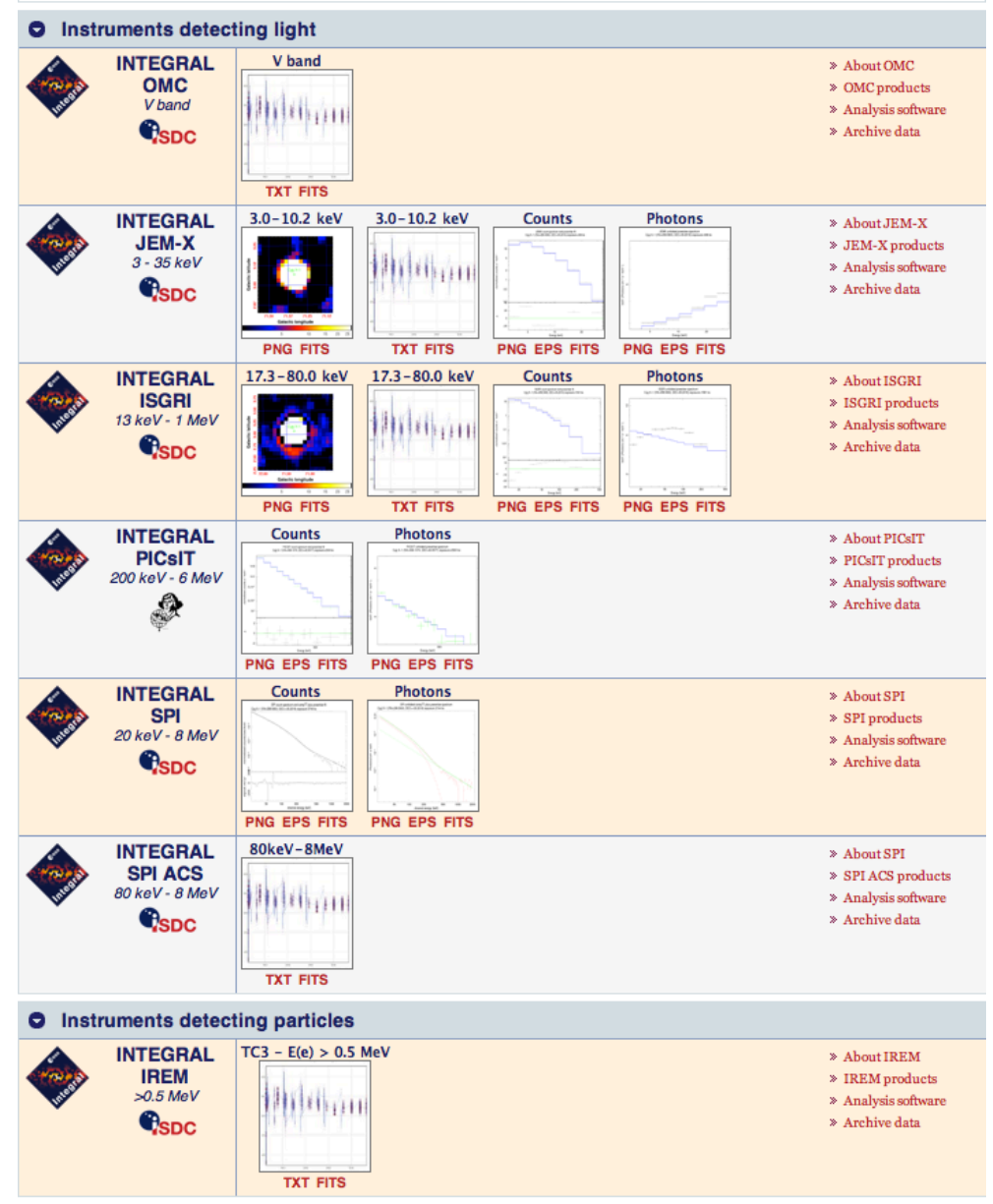

Figure 1: The HEAVENS query interface allows to define source name/sky position, set of time intervals and instrument specific parameters. The query results, displayed as icons can be stored in various formats or opened in specific windows. 
in shape. As the current ISGRI OSA requires a time dependent database of ancillary spectral responses, a weighted averaged spectral response is built corresponding to the user specified set of time intervals and delivered together with each extracted spectrum.

The pixel binary files structure and disk organization has been optimized for disk access speed. The extraction software has been optimized to minimize computational time. Generating products for the full mission takes of the order of a minute, even in the central regions of the galaxy, that accumulate close to $10^{4}$ pointings per pixels. The JEM-X pixel database includes data from both JEM-X units. The data are combined/averaged when presented to the user.

The ISGRI and JEM-X pixel data that have a maximum time resolution of one pointing (i.e. about $20 \mathrm{~min}$.), will be augmented by high time resolution lightcurves for all sources bright enough or featuring bright flares. In all these cases lightcurves with a time resolution adapted to the source count rate will be generated and provided together with the pixel based data transparently.

\subsection{PICsIT products}

Mission averaged PICsIT [3, 6] spectra are provided in HEAVENS for a small number of sources bright above $100 \mathrm{keV}$. The spectra have been generated by the method described in [7].

A study is on-going to assess the possibility to provide a pixel based PICsIT archive similar to that build for ISGRI and JEM-X, as described above. Indeed the method used for ISGRI and JEMX can be applied to any set of sky images that have proper statistical uncertainty. Unfortunately the current PICsIT images still have large systematics that are not averaged out by accumulation. The variance image are not describing correctly these uncertainties. When these systematic features will be corrected for (both at the detector and sky image level), PICsIT data will benefit from the same infrastructure used for ISGRI and JEMX and HEAVENS will provide images, lightcurves and spectra up to the $\mathrm{MeV}$ range.

\subsection{SPI products}

HEAVENS includes the SPI spectra of 25 of the sources listed in [8], for which a flux $f(25-$ $50 \mathrm{keV})>20 \mathrm{mCrab}$ is reported. The HEAVENS spectra have been obtained with the SPIMODFIT analysis module included in the OSA 9 release, and cover a wide energy range, between $25 \mathrm{keV}$ to $1 \mathrm{MeV}$. Data selection includes the pointing mode, a minimum SPI observation time in each Science Window (SCW) $\geq 500 \mathrm{~s}$ and an offset ranging between 4 and $12 \mathrm{deg}$ of the source position with respect to the SPI pointing direction.

During the analysis procedure, some non-default parameter settings have been used for all sources, which include a 1-day background time variability coefficient (which takes also into account the annealing periods of SPI) and a minimum flux of $10^{-6}$ counts s ${ }^{-1}$ in the catalogue extraction step (see the SPI user manual ${ }^{2}$ for more details). In addition, some key parameters have been defined for each source (e.g. the spectral binning) and, in order to improve the final results, also a case-by-case spectral fitting modelisation in XSPEC (e.g. cyclotron lines) has been applied. The SPI catalogue will be extended to provide lightcurves and spectral timing information for about 90 sources with a timing resolution of a few days.

\footnotetext{
${ }^{2}$ available at http://www.isdc.unige.ch/integral/analysis
} 


\subsection{OMC lightcurves}

HEAVENS offers also access to optical data (Johnson's V band) from INTEGRAL's Optical Monitoring Camera OMC [9]. The OMC transmits data for sources selected in a predefined catalogue [10]. The OMC light curves have been generated with OSA software. V magnitudes are derived from counts gathered in 3 by 3 CCD pixel square windows.

HEAVENS presents all data points for which the magnitude could be derived and which did not encounter severe problems due to position inaccuracy, invalidating the observed flux (PROBLEMS flag < 8192). The user can filter these data further according to the extraction quality. The most restrictive option ("good") provides only data points with no problem at all; the "medium" option adds data that encountered some problems during computation of the position centroid or of the PSF, or for which the magnitude value had to be extrapolated. These problems cause usually minor inaccuracies of the magnitude and flux, and often reflect physical features of the data (e.g. close companions, extended source). The "all data" option includes also data affected e.g. by bad pixels or large sky background (PROBLEMS flag from 128 to 4096). The data are offered both in magnitude form and as a $v F_{V}\left(=5500 \times 3.64 \times 10^{-9} \mathrm{erg} \mathrm{cm}^{-2} \mathrm{~s}^{-1} \AA^{-1} \times 10^{-0.4 m_{V}}\right)$ flux [11].

\subsection{SPI-ACS and IREM lightcurves}

The camera of SPI is shielded on the side walls and rear side by a large anticoincidence shield (ACS). Apart from it's primary purpose of protecting the SPI camera, the ACS provides a large effective area (up to $8000 \mathrm{~cm}^{2}$ ) for the detection of bursts ([12]), but with no positional or energy information. The ACS consists of 91 BGO crystals monitored by 91 photomultipliers, which are read out by 91 front-end electronic (FEE) boxes. The low-energy thresholds in the FEEs were set to approximately $75_{-25}^{+75} \mathrm{keV}$ (they extend over a wide energy range and are not sharp). As a veto shield the ACS has no upper limit of the energy range.

The ACS detects about 300 GRBs or GRB candidates per year, though many of them are not triggered by the IBAS. Sensitivity of the ACS depends on the GRB arrival direction with respect to the satellite and it's spectrum, but can be roughly estimated to be about $\sim 10^{-6} \mathrm{erg} / \mathrm{s} \mathrm{cm}^{2}$ for the minimal $50 \mathrm{~ms}$ time bins and $\sim 10^{-7} \mathrm{erg} / \mathrm{s} \mathrm{cm}^{2}$ for a $1 \mathrm{~s}$ integration. The ACS overall veto count rate is provided in HEAVENS for the full mission with a resolution of $50 \mathrm{~ms}$.

IREM detector lightcurves are available for 15 particle energy channels and for the full mission with a resolution of about 1 minute. They allow to monitor the particle rate along the orbit and to map the structure of the radiation belts close to perigee.

\section{Outlook}

The INTEGRAL data in HEAVENS will be regularly completed by new data becoming publicly available, extended with better data for the SPI and PICsIT instruments and extended with high time resolution ISGRI and JEM-X data for bright sources.

A new interface is also being developed for outreach purposes and data from other high-energy missions will be made available. After some months in operations, HEAVENS has already doubled the number of visits to the INTEGRAL archive. Providing a seamless access to INTEGRAL data allows scientists to make the best use of the mission products and will soon allow the public to get a glimpse of high-energy astrophysics data, methods and results. 


\section{Acknowledgments}

AF was supported by the Polish MNiSW grant 362/1/N-INTEGRAL (2009-2012).

\section{References}

[1] C. Winkler et al. The INTEGRAL mission. A\&A, 411-L1, 2003.

[2] T. Courvoisier et al. The INTEGRAL Science Data Centre. A\&A , 411-53, 2003.

[3] P. Ubertini. IBIS: The Imager on-board INTEGRAL. A\&A , 411-L131, 2003.

[4] F. Lebrun. ISGRI: The INTEGRAL Soft Gamma-Ray Imager. A\&A , 411-L141, 2003.

[5] N. Lund et al. JEM-X: The X-ray monitor aboard INTEGRAL. A\&A , 411-L231, 2003.

[6] G. Di Cocco et al. BIS/PICsIT in-flight performances. A\&A , 411-L189, 2003.

[7] P. Lubinski. Analysis of extremely low signal-to-noise ratio data from INTEGRAL/PICsIT. A\&A, 496-557, 2009.

[8] L. Bouchet et al. INTEGRAL SPI All-Sky View in Soft Gamma Rays: A Study of Point-Source and Galactic Diffuse Emission. ApJ , 679-1215, 2008.

[9] J.M. Mas-Hesse et al. OMC: An Optical Monitoring Camera for INTEGRAL. Instrument description and performance. A\&A , 411-L261, 2003.

[10] A. Domingo et al. The Input Catalogue for the OMC camera onboard INTEGRAL. A\&A , 411-L281, 2003.

[11] W. Wamsteker et al. Standard stars and calibration for JHKLM photometry. A\&A , 97-329, 1981.

[12] A. von Kienlin et al. Integral spectrometer spi's grb detection capabilities. grbs detected inside spi's fov and with the anticoincidence system acs. A\&A, 411-299, nov 2003. 